\title{
Cooperation of Hot Holes and Surface Adsorbates in Plasmon- Driven Anisotropic Growth of Gold Nanostars
}

\author{
Wenxiao Guo, ${ }^{\dagger}$ Aaron C. Johnston-Peck, ${ }^{\ddagger}$ Yuchao Zhang, ${ }^{\dagger}, \S$ Yue Hu,${ }^{\dagger}$ Jiawei Huang, ${ }^{\dagger}$ and \\ Wei David Wei ${ }^{\dagger *}$ \\ 'Department of Chemistry and Center for Catalysis, University of Florida, Gainesville, Florida 32611, United \\ States \\ Material Measurement Laboratory, National Institute of Standards and Technology, 100 Bureau Drive, \\ Gaithersburg, Maryland 20899, United States \\ ${ }^{\S}$ Present address: Key Laboratory of Photochemistry, CAS Research/Education Center for Excellence in \\ Molecular Sciences, Institute of Chemistry, Chinese Academy of Sciences, Beijing 100190, P. R. China \\ *Address correspondence to wei@chem.ufl.edu
}

\section{Experimental Procedures}

\section{Materials}

Gold (III) chloride trihydrate $\left(\mathrm{HAuCl}_{4}\right)$, polyvinylpyrrolidone $(\mathrm{PVP}, \mathrm{Mw}=40,000 \mathrm{Da})$, and potassium iodide (KI) were purchased from Sigma Aldrich. Methanol (99.8\%) was obtained from Fisher Chemicals. All synthesis processes were performed with Nanopure water (18.2 M $\Omega)$. All glassware was cleaned with aqua regia solution and rinsed thoroughly with Nanopure water before use (caution! Aqua regia is highly corrosive and toxic: handle with care and use appropriate personal protection equipment).

\section{Instrumentation}

The light source used for the plasmon-driven growth of Au nanostars was a halogen lamp (Dolan-Jenner MI-150) equipped with an internal IR holder/filter (to avoid excessive heating). Single-wavelength bandpass filters were purchased from Thorlabs. Ultravioletvisible-near infrared (UV-Vis-NIR) spectra of products were collected using a Shimadzu UV-2600 spectrophotometer. Scanning electron microscopy (SEM) images of $\mathrm{Au}$ nanostars were collected using a FEI Nova Nano 430 SEM at the Nanoscale Research Facility of the University of Florida. Selected area electron diffraction (SAED) patterns and high-angle annular dark-field (HAADF) scanning transmission electron microscopy (STEM) images were acquired from a transmission electron microscope (TEM) operated 
at an accelerating voltage of $300 \mathrm{kV}$ at Material Measurement Laboratory of National Institute of Standards and Technology (Note: Certain commercial equipment, instruments, or materials are identified in this paper in order to specify the experimental procedure adequately. Such identification is not intended to imply recommendation or endorsement by the National Institute of Standards and Technology, nor is it intended to imply that the materials or equipment identified are necessarily the best available for the purpose.). The probe forming optics of the instrument was aberration-corrected, and the convergence angle was set to approximately $13.5 \mathrm{mrad}$. HAADF-STEM images were acquired using a Fischione Model 3000 detector, in which the inner collection angle was set to approximately either $58 \mathrm{mrad}$ or $71 \mathrm{mrad}$. Electrochemical experiments were conducted using an Autolab potentiostat (PGSTAT302N) from Metrohm. Light sources used in electrochemical experiments were single-wavelength LEDs (470 $\mathrm{nm}$ and $530 \mathrm{~nm}$ ) with a maximum power density of $240 \mathrm{~mW} / \mathrm{cm}^{2}$.

\section{Experimental details}

\section{Synthesis of Au nanostars:}

$\mathrm{Au}$ seeds were synthesized using a previously reported method. ${ }^{1,2}$ The seed solution was aged for more than two weeks before use. To synthesize Au nanostars, a solution containing $5 \mathrm{~mL}$ of $\mathrm{H}_{2} \mathrm{O}, 500 \mu \mathrm{L}$ of $5 \mathrm{mg} / \mathrm{mL}$ PVP solution, $500 \mu \mathrm{L}$ of methanol, $400 \mu \mathrm{L}$ of $10 \mathrm{mM} \mathrm{HAuCl}_{4}$ solution, and $1 \mu \mathrm{L}$ of aged seed solution was prepared (i.e., growth solution). The seed solution contained a mixture of planar-twinned seeds and pentatwinned seeds, while only planar-twinned seeds can lead to the formation of Au nanostars. ${ }^{1}$ To isolate planar-twinned Au seeds from the mixture, the growth solution was incubated in a water bath at $24.5^{\circ} \mathrm{C}$ under the dark condition for $24 \mathrm{~h}$. Penta-twinned Au seeds were reported to grow faster in the dark and would gain a heavier mass after the incubation. ${ }^{1}$ The incubated growth solution was then centrifuged at 10,000 rpm for $10 \mathrm{~min}$, allowing for the precipitation of penta-twinned seeds. The supernatant of the growth solution after the centrifugation contained mainly planar-twinned seeds and was then transferred to a glass vial with a Teflon-lined cap for the plasmon-driven growth. The growth solution was irradiated by a halogen lamp equipped with a $520 \pm 10 \mathrm{~nm}$ bandpass filter at $3.4 \mathrm{~mW} / \mathrm{cm}^{2}$. 
After 20 min of irradiation, $20 \mu \mathrm{L}$ of $1 \mathrm{mM} \mathrm{KI}$ solution was injected into the growth solution (overall KI concentration: $4 \mu \mathrm{M}$ ). The solution was then irradiated for another 60 min to complete the growth. The growth in the dark was carried out by storing the incubated and centrifuged growth solution in the dark at $50{ }^{\circ} \mathrm{C}$ for $24 \mathrm{~h}$.

\section{Wavelength-dependent growth}

Wavelength-dependent growth was carried out after the incubation and centrifugation of a growth solution. It should be noted that small planar-twinned Au seeds in the growth solution only had strong absorption near $520 \mathrm{~nm},{ }^{1}$ so that the growth would not happen if the growth solution was irradiated with wavelengths too far from $520 \mathrm{~nm}$ from the beginning of plasmon-driven synthesis. Instead, the growth solution was first irradiated at $520 \pm 10 \mathrm{~nm}$ for $20 \mathrm{~min}$ in the absence of iodide. This initial growth step enabled planartwinned $\mathrm{Au}$ seeds to develop into anisotropic (prism-like) structures that exhibited a broader SPR absorption band. ${ }^{1}$ After this initial growth, KI was added to the growth solution, and the mixture was irradiated using bandpass filters with targeting wavelength (from $460 \mathrm{~nm}$ to $640 \mathrm{~nm}$ ) continuously for another $60 \mathrm{~min}$.

\section{Electrochemical measurements:}

Electrochemical measurements were carried out in a three-electrode electrochemical cell with a Pt wire counter electrode and a $\mathrm{Ag} / \mathrm{AgCl}(3.5 \mathrm{M} \mathrm{KCl})$ reference electrode. The working electrode was a Au nanocrystal electrode, which was fabricated by sputtering 10 $\mathrm{nm}$ of $\mathrm{Au}$ film on a FTO electrode and then calcinating for $2 \mathrm{~h}$ at $600{ }^{\circ} \mathrm{C}$. All electrochemical experiments were conducted in $50 \mathrm{~mL}$ of $0.5 \mathrm{M} \mathrm{NaClO}_{4}$ with a $\mathrm{pH}$ value tuned to 3.0 using $\mathrm{HClO}_{4}$ (i.e., the $\mathrm{pH}$ of the growth solution). To modify the $\mathrm{Au}$ nanocrystal electrode with iodide, the working electrode was immersed in a $0.5 \mathrm{M} \mathrm{NaClO}_{4}$

solution containing KI until its rest potential became stable. The PVP-adsorbed electrode was prepared by immerging a Au nanocrystal electrode in a $20 \mathrm{mg} / \mathrm{mL}$ PVP solution overnight and then rinsing with water.

\section{Edge facets identification using STEM:}

Dodecagonal intermediates obtained during the growth of Au nanostars were isolated from the growth solution and loaded on a $\mathrm{C}$-supported $\mathrm{Cu}$ grid. Amorphous silica particles 
were deposited prior to the deposition of Au nanostars to tilt Au nanostars for imaging their edges. As shown in Figure S1, the microscope stage was tilted in the STEM chamber to orient the nanostructure to an "edge-on" pattern, in which the edge of interest was aligned with the incident electron beam. The obtained STEM images were filtered using a "Trend Substrate" filter (HREM-Filters Lite version 3.0, HREM Research Inc.) as implemented in Digital Micrograph (Gatan Inc.) to enhance the visualization of atomic columns. The filter removed the low-frequency component of the image (background) that was prominent due to the combination of a high atomic number and a large sample thickness. Facets exposed on edges were then identified by matching the arrangement of atoms with that of characteristic crystal models.
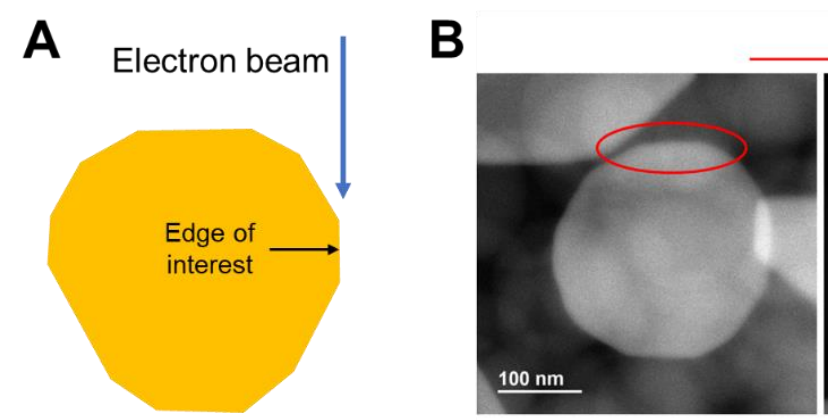

Tilting

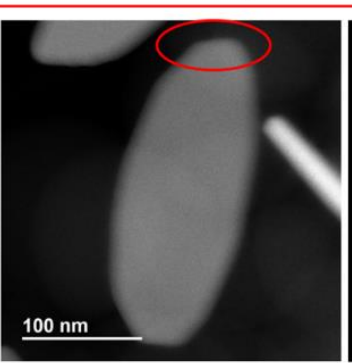

Figure S1. The process of obtaining the atomic-resolved STEM image of an edge of a dodecagonal structure. (A) A schematic showing the orienting of Au nanostructures for measuring edge facets. The sample stage was tilted until the edge of interest aligned with the incident electron beam (blue arrow). (B) An example of the tilting process to align a nanostructure. The red circle indicated the edge to be imaged. 


\section{Supplementary Figures and Tables}
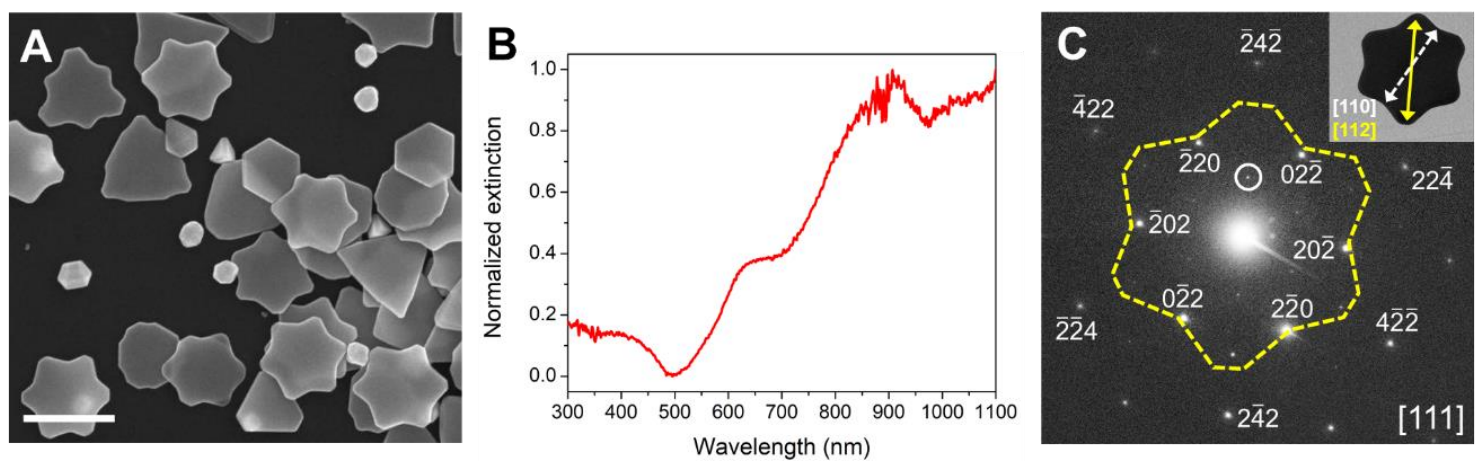

Figure S2. Characterizations of Au nanostars. (A) A low-magnification SEM image of Au nanostars. The scale bar represented $500 \mathrm{~nm}$. (B) The UV-Vis spectrum obtained from an aqueous suspension of $\mathrm{Au}$ nanostars. The peak at ca. $900 \mathrm{~nm}$ stemmed from the $\mathrm{Au}$ nanostars, and the shoulder peak at ca. $600 \mathrm{~nm}$ originated from the small population of $\mathrm{Au}$ NPs grown from the penta-twinned $\mathrm{Au}$ seeds that remained in the growth solution after incubation and centrifugation. (C) A SAED pattern collected from a $\mathrm{Au}$ nanostar (corresponding TEM image shown in the inset) lying flat on a $\mathrm{Cu}$ grid. The pattern was indexed to a [111] zone axis, suggesting that the top and bottom surfaces of the nanostar are terminated by $\{111\}$ facets, which was confirmed by atomic resolution HAADF-STEM imaging (not shown). $\{422\}$ and $\{220\}$ Bragg reflections were labelled. A forbidden $\frac{1}{3}\{422\}$ Bragg reflection marked by the white circle indicated the presence of planar defects. ${ }^{3-6}$ Crystallographic directions within Au nanostars were extracted from the SAED pattern. As indicated in the inset TEM image, tips of Au nanostars grew along [112] directions (yellow solid arrows), and trenches grew along [110] directions (white dashed arrows). Only one pair of tips and trenches were labeled for a clearer view. 

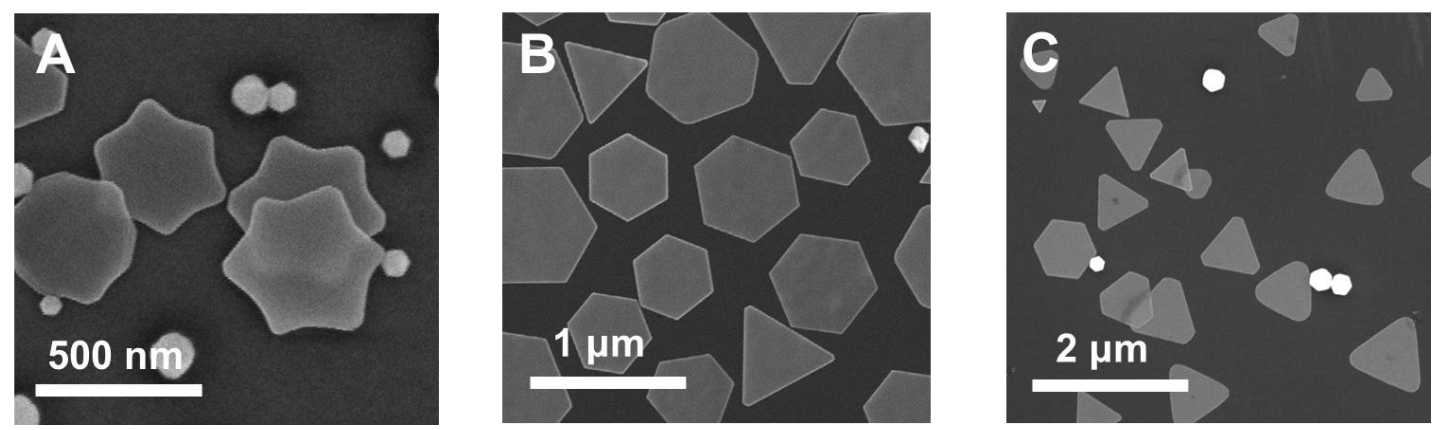

Figure S3. SEM images of $\mathrm{Au}$ nanostructures formed under different experimental conditions. (A) Au nanostars formed from the plasmon-driven growth in the presence of 4 $\mu \mathrm{M}$ of NaI. (B) Regular nanoprisms formed from the plasmon-driven growth in the absence of iodide. (C) Regular nanoprisms formed from the growth in the dark in the presence of iodide.
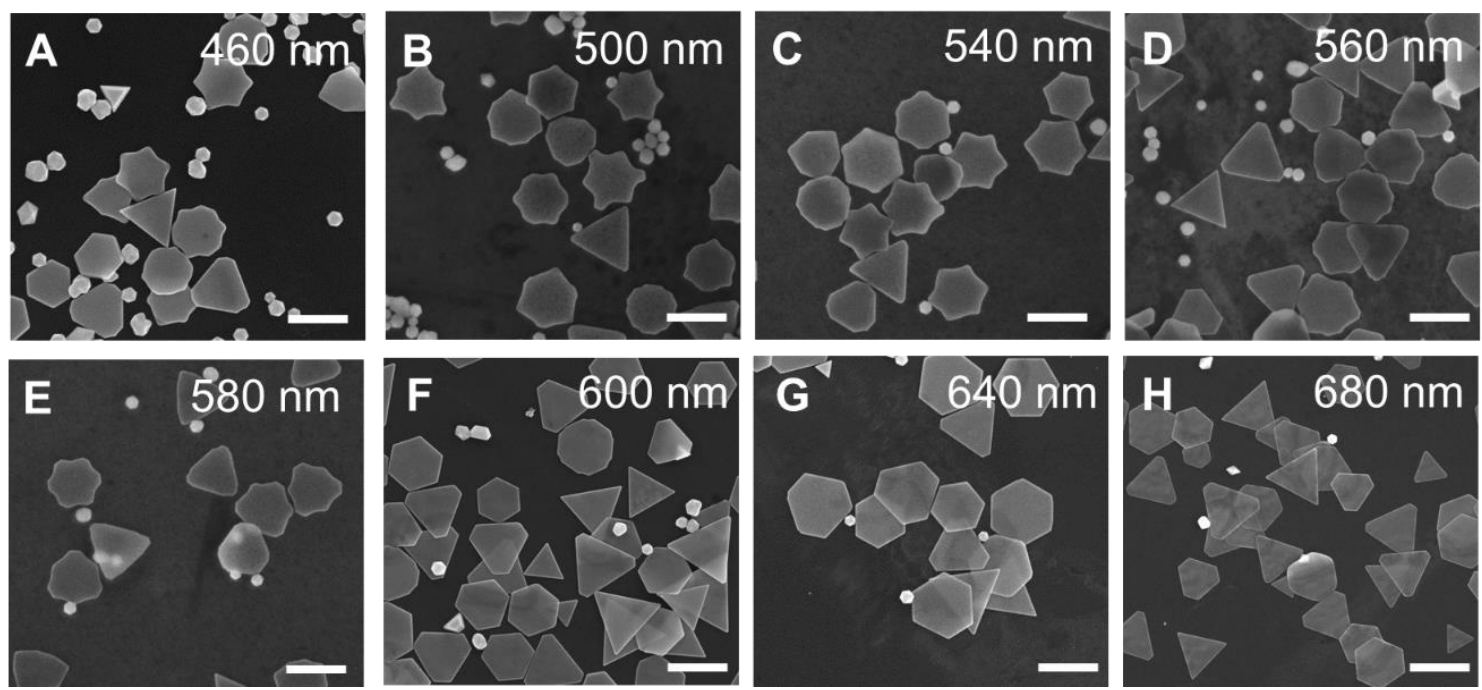

Figure S4. SEM images of Au nanostructures formed from the plasmon-driven growth with different irradiation wavelengths. (A) - (E) Au nanostars formed when shorter wavelengths $\left(<600 \mathrm{~nm}, 3.4 \mathrm{~mW} / \mathrm{cm}^{2}\right)$ were used. $(\mathrm{F})-(\mathrm{H})$ Regular Au nanoprisms formed when longer wavelengths $\left(\geqslant 600 \mathrm{~nm}, 3.4 \mathrm{~mW} / \mathrm{cm}^{2}\right)$ were used. Scale bars stand for 500 $\mathrm{nm}$ in all figures. 


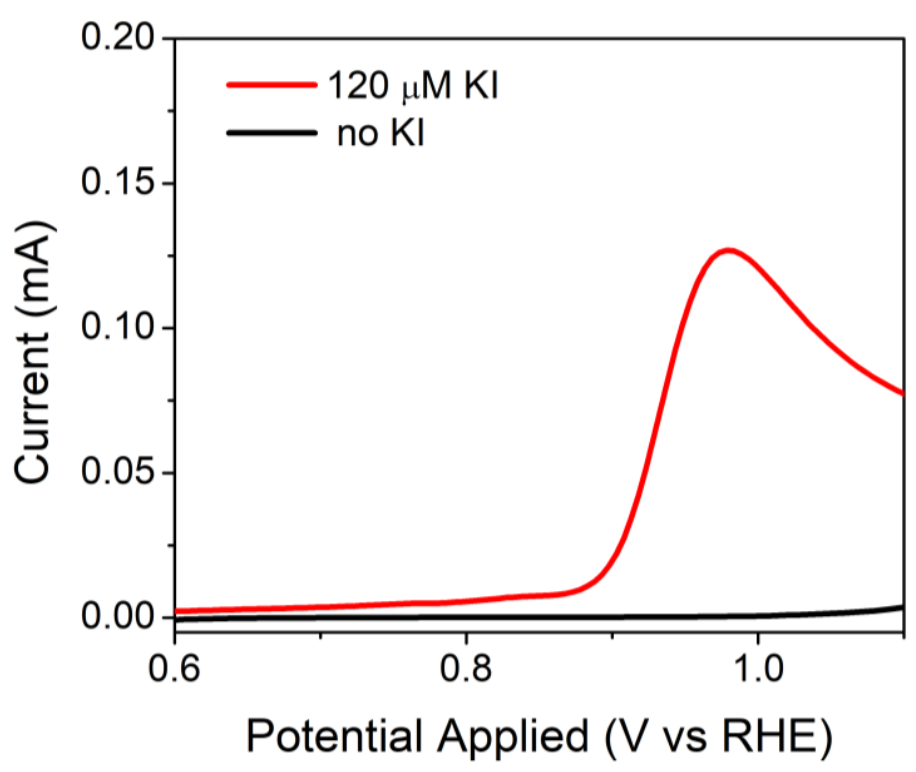

Figure S5. A linear sweep voltammetry (LSV) scan obtained on Au nanocrystal electrodes in the presence (red curve) and absence (black curve) of $120 \mu \mathrm{M} \mathrm{KI}$. A peak at ca. $0.95 \mathrm{~V}$ vs. RHE was observed for the iodide-adsorbed electrode, which originated from the oneelectron oxidation of $\mathrm{Au}^{0}$ to $\mathrm{AuI}_{2}^{-}{ }^{-7}$ 

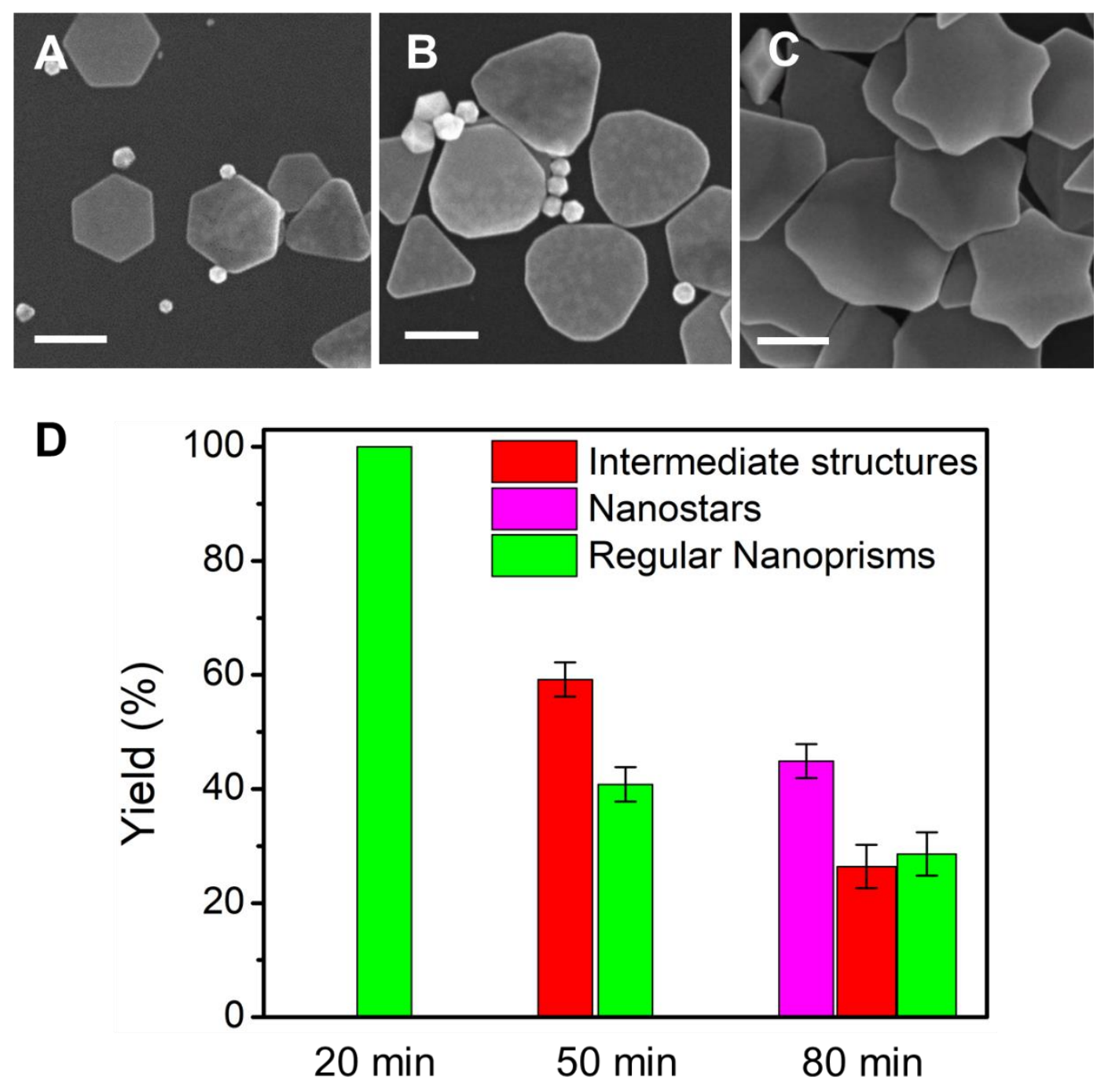

Figure S6. SEM images showing the ensemble of Au nanostructures during the plasmondriven growth in the presence of iodide after (A) 20 minutes, (B) 50 minutes, and (C) 80 minutes of growth. (D) A statistical analysis of products formed at each stage. The yield of dodecagonal intermediate structures at 50 min was $59 \pm 3 \%$, and the yield of nanostars at 80 min was $45 \pm 3 \%$. Over 200 nanostructures were counted at each stage to obtain the distribution of structures. Scale bars in all (A), (B), and (C) stand for $200 \mathrm{~nm}$. 
A

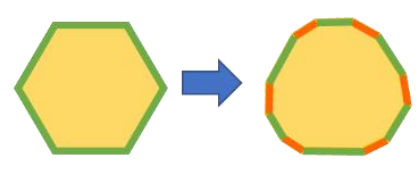

B

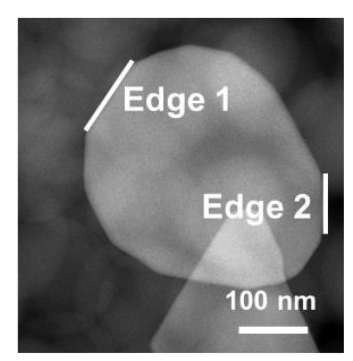

\section{c}

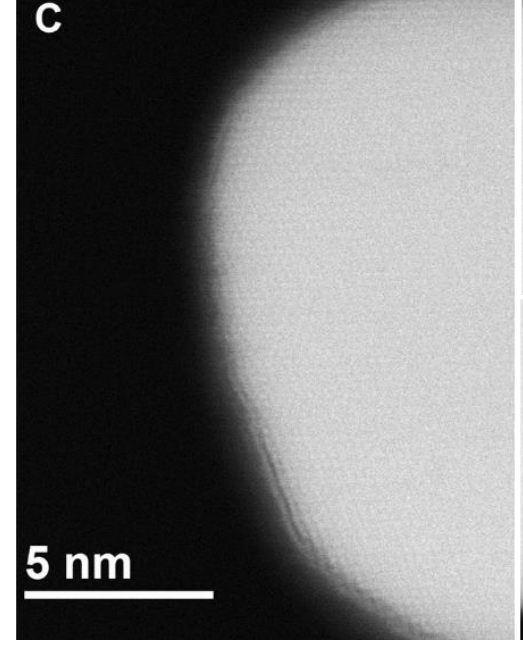

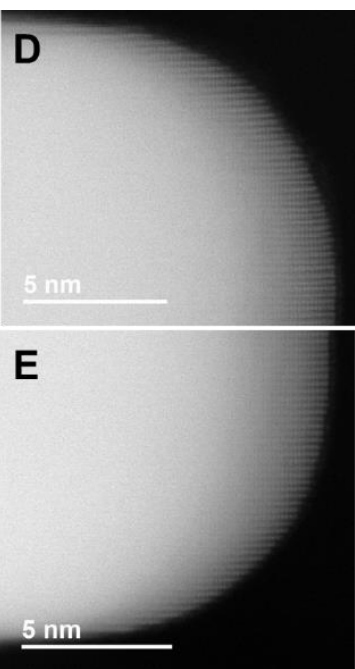

Figure S7. Facet identification of the intermediate structure formed during the growth of Au nanostars. (A) A schematic showing the truncation of a regular Au nanoprism to form the dodecagonal intermediate. Edges marked with green color were composed of low-index facets that are typical for Au nanoprisms, and edges marked with orange color were newly formed intersecting edges with high-index facets. Two types of edges were arranged alternatively. (B) Indication of the position of two edges on an individual dodecagonal intermediate structure examined by STEM. By arrangement, edge 1 and edge 2 represented those two types of edges shown in (A). (C) HAADF-STEM images along edge 1 of the intermediate dodecahedral structure. (D) and (E) HAADF-STEM images along edge 2 of the intermediate dodecahedral structure. (C), (D), and (E) are unfiltered copies corresponding to images shown in Figure 4A, 4B, and 4C of the main text, respectively. From the facet identification shown in Figure 4, it can be concluded that edge 1 corresponded to a low-index green edge in (A), and edge 2 corresponded to a high-index orange edge in $(\mathrm{A})$.

The facet analysis was conducted on 13 edges from 10 dodecagonal structures. Two types of edges with drastically different percentages of low-index regions (i.e., $\mathrm{Au}\{111\},\{110\}$, and $\{100\}$ ) and high-index regions were identified (Table S1). The first type of edges had approximately $68 \%$ low-index components, while the second type of edges only had about $23 \%$ low-index component. Edges with more high-index components corresponded to active sites for hot-hole-induced etching of $\mathrm{Au}^{0}$. 
Table S1. Summary of edge facets of dodecagonal intermediates

\begin{tabular}{|c|c|c|}
\hline Structure \# & Low-index component (\%) & High-index ratio component (\%) \\
\hline \multicolumn{3}{|c|}{ Type 1 } \\
\hline 1 & 62.5 & 37.5 \\
\hline 2 & 68.9 & 31.1 \\
\hline 3 & 66.1 & 33.9 \\
\hline 4 & 72.8 & 27.2 \\
\hline 5 & 74.3 & 25.7 \\
\hline 6 & 71.3 & 28.7 \\
\hline 7 & 69.4 & 30.6 \\
\hline 8 & 65.5 & 34.5 \\
\hline 9 & 63.8 & 36.2 \\
\hline 10 & 66.6 & 33.4 \\
\hline Average & $68.1 \pm 3.9$ & $31.9 \pm 3.9$ \\
\hline & & Type 2 \\
\hline 11 & 20.8 & 79.2 \\
\hline 12 & 20.0 & 76.8 \\
\hline 13 & 28.7 & 4.8 \\
\hline Average & $23.2 \pm 4.8$ & \\
\hline
\end{tabular}




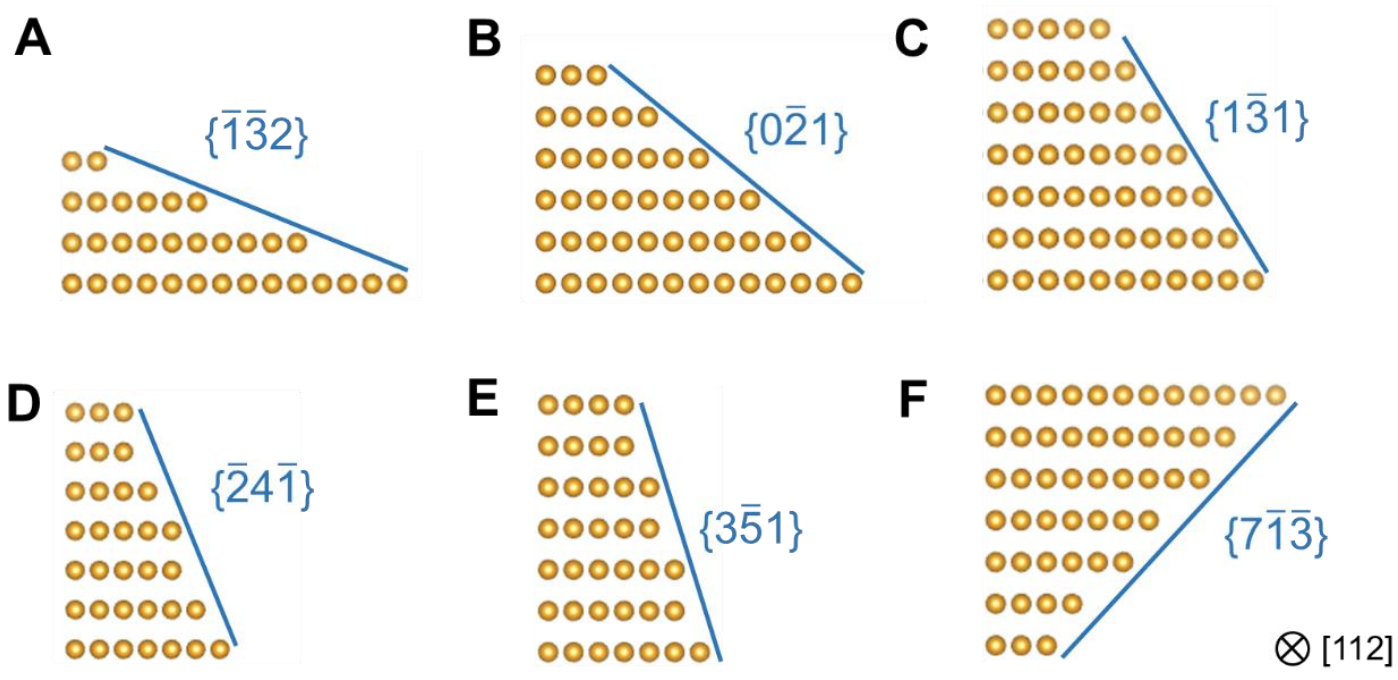

Figure S8. Schematics of high-indexed facets observed on dodecagonal intermediates as viewed along the [112] direction.
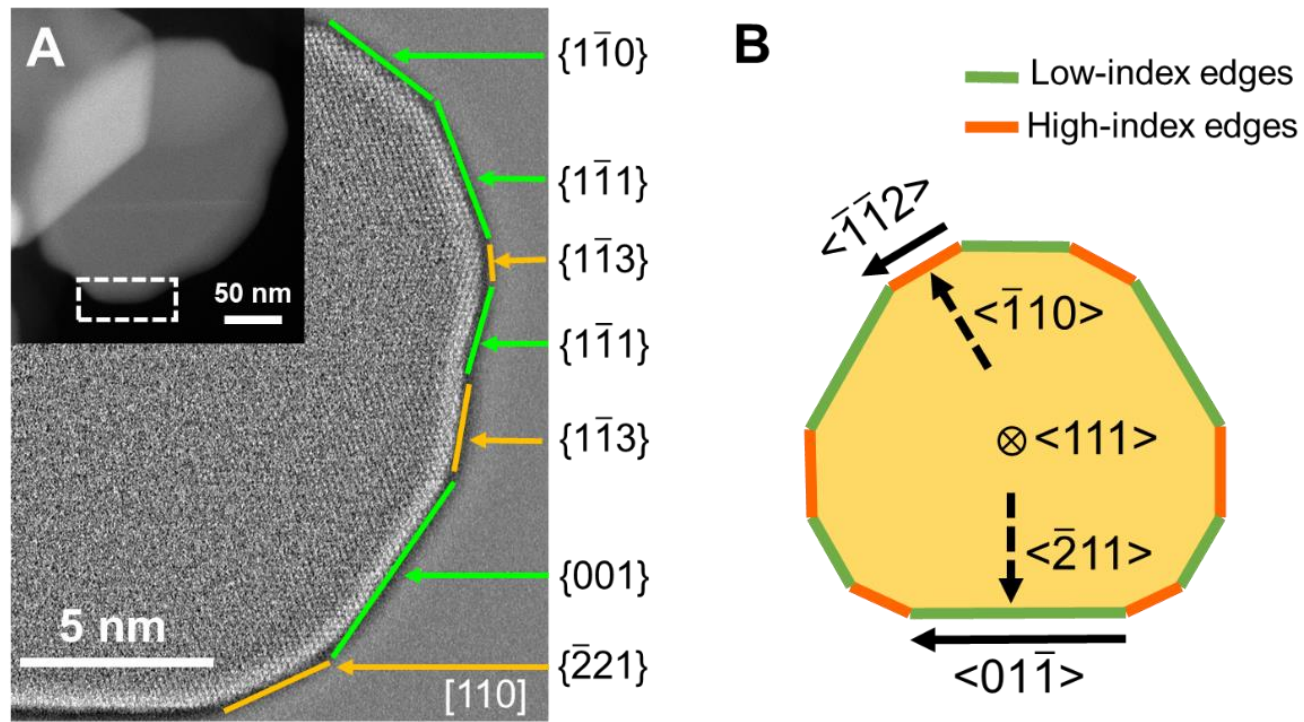

Figure S9. Growth of dodecagonal intermediate nanostructures into Au nanostars. (A) A HAADF-STEM image of an early-stage nanostar's tip (indicated by the dashed frame). The tip mainly consisted of low-index facets, which indicated the faster growth of edges dominated by low-index facets of intermediate structures, confirming that tips of $\mathrm{Au}$ 
nanostars evolved from low-index edges of dodecagonal intermediate nanostructures. (B) Derived crystallographic directions within a dodecagonal intermediate nanostructure. As indicated in Figure 4 in the main text, zone axes used to obtain HAADF-STEM images on a low-index edge and a high-index edge were [110] and [112] (i.e., paralleled to chosen edges), respectively. Since the top and bottom faces of those dodecagonal intermediate nanostructures were bound by $\{111\}$ facet, it can be derived from the crystal structure of $\mathrm{Au}$ that low-index edges grew along [112] directions and high-index edges grew along [110] directions, which matched with growth direction of tips (i.e., [112]) and trenches (i.e., [110]) of nanostars (Figure S2B), respectively. This match in growth directions indicated that tips of nanostars evolved from low-index facets and trenches evolved from high-index facets.
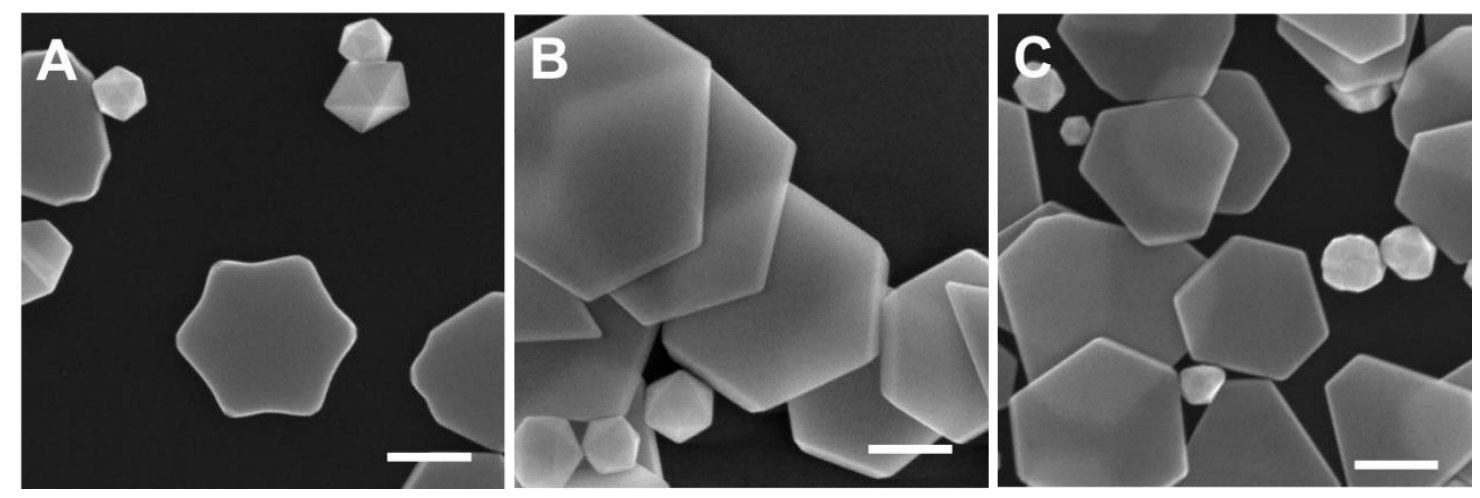

Figure S10. SEM images showing the plasmon-driven synthesis of Au nanostars in the presence of $\mathrm{KBr}$. (A) The formation of Au nanostars in the presence of $0.2 \mathrm{mM} \mathrm{KBr}$ under $460 \mathrm{~nm}$ irradiation. (B) The formation of regular Au nanoprisms in the presence of $4 \mu \mathrm{M}$ $\mathrm{KBr}$ under $460 \mathrm{~nm}$ irradiation. (C) The formation of regular Au nanoprisms in the presence of $0.2 \mathrm{mM} \mathrm{KBr}$ under $560 \mathrm{~nm}$ irradiation. It can be seen that to obtain Au nanostars using bromide, a higher concentration of $\mathrm{KBr}$ and the shorter-wavelength irradiation that directly caused photoexcitation of Au (i.e., interband transitions) to form more d-band holes should be used. The scale bar stands for $200 \mathrm{~nm}$ in all images. 

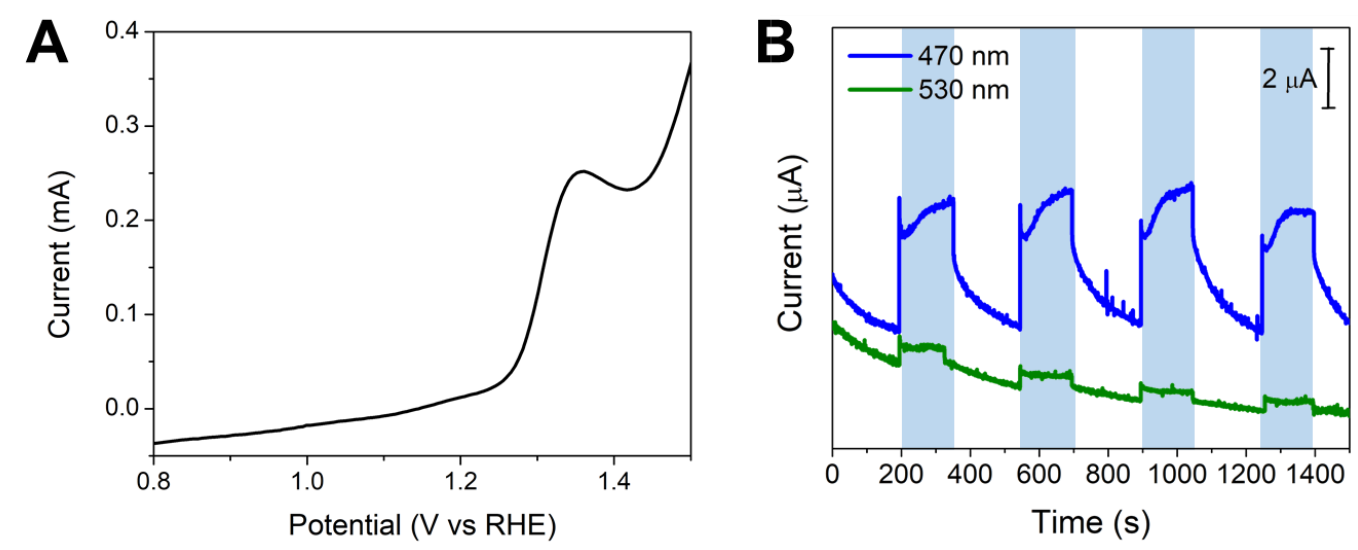

Figure S11. Photoelectrochemical results confirming the hot-hole-driven etching of $\mathrm{Au}^{0}$ assisted by bromide. (A) A LSV scan obtained on a Au nanocrystal electrode in the presence of $\mathrm{KBr}$. A peak at ca. $1.35 \mathrm{~V}$ vs. RHE stood for the one-electron oxidation of $\mathrm{Au}^{0}$ to $\mathrm{AuBr}_{2}{ }^{-7}$ (B) I-t curves collected at $1.40 \mathrm{~V}$ vs. RHE showing the anodic photocurrent of a $\mathrm{Au}$ electrode in the presence of bromide. Shaded regions in (B) represented the light irradiation using either $530 \mathrm{~nm}$ (green curve) or $470 \pm 10 \mathrm{~nm}$ (blue curve) LED light source $\left(240 \mathrm{~mW} / \mathrm{cm}^{2}\right)$. A significant enhancement can be observed only when using the $470 \mathrm{~nm}$ irradiation.

\section{References}

(S1) DuChene, J. S.; Niu, W.; Abendroth, J. M.; Sun, Q.; Zhao, W.; Huo, F.; Wei, W. D. Halide anions as shape-directing agents for obtaining high-quality anisotropic gold nanostructures. Chem. Mater. 2012, 25 (8), 1392-1399.

(S2) Zhai, Y.; DuChene, J. S.; Wang, Y. C.; Qiu, J.; Johnston-Peck, A. C.; You, B.; Guo, W.; DiCiaccio, B.; Qian, K.; Zhao, E. W.; Ooi, F.; Hu, D.; Su, D.; Stach, E. A.; Zhu, Z.; Wei, W. D. Polyvinylpyrrolidone-induced anisotropic growth of gold nanoprisms in plasmon-driven synthesis. Nat. Mater. 2016, 15 (8), 889-895.

(S3) Langille, M. R.; Personick, M. L.; Mirkin, C. A. Plasmon-Mediated Syntheses of Metallic Nanostructures. Angew. Chem., Int. Ed. 2013, 52 (52), 13910-13940.

(S4) Kirkland, A. I.; Jefferson, D. A.; Duff, D. G.; Edwards, P. P.; Gameson, I.; Johnson, B. F. G.; Smith, D. J. Structural studies of trigonal lamellar particles of gold and silver. Proc. R. Soc. A 1993, 440 (1910), 589-609.

(S5) Carim, A. H.; Lew, K.-K.; Redwing, J. M. Bicrystalline silicon nanowires. Adv. Mater. 2001, 13 (19), 1489-1491. 
(S6) Germain, V.; Li, J.; Ingert, D.; Wang, Z. L.; Pileni, M. P. Stacking faults in formation of silver nanodisks. J. Phys. Chem. B 2003, 107 (34), 8717-8720.

(S7) Aylmore, M. G. Chapter 27 - Alternative lixiviants to cyanide for leaching gold ores. In Gold Ore Processing, second edition, Adams, M. D., Ed.; Elsevier: Cambridge, MA, 2016; pp 447-484. 